\title{
Survey of Prevalence and Attitudes of Health-Related Self-Help Approaches Among Older Adults
}

\author{
Angela Drent ${ }^{1}$ and Manoj Sharma ${ }^{2}$ \\ ${ }^{1}$ Community Networking / 55Plus, Methodist Health System \\ ${ }^{2}$ University of Cincinnati
}

\begin{abstract}
The purpose of this study was to determine the prevalence and determinants of health-related self-help approaches among older adults. A cross-sectional survey of community members over 55 years of age enrolled in a health-related program in the Midwestern United States was conducted. Data pertaining to usage, knowledge, costs, self-efficacy, and expectations of four commonly used self-help approaches comprising of self-prayer, relaxation, yoga, and tai chi was collected for older adults. A total of 2,726 (26.4\%) respondents completed and returned the questionnaire. Self-prayer was reported as the most common self-help approach with $60 \%$ usage. Mean knowledge score was found to be 4.46 units and selfefficacy 4.39 units both in a possible range of 0-12 units. Using logistic regression modeling, knowledge, self-efficacy and expectations were found to be significant predictors for respondents who were in preparation, action or maintenance stages as opposed to pre-contemplation or contemplation. Educational programs that build knowledge, self-efficacy and expectations about self-help approaches among older adults are needed.
\end{abstract}

(c) 2006 Californian Journal of Health Promotion. All rights reserved.

Keywords: Complimentary, alternative, self-help, older adults

\section{Introduction}

Different systems of medicine and health have been widely used all over the world. The predominant system of medicine that has grown in the West is often considered a mainline system. However, there are several other systems of medicine and health that are slowly gaining recognition in the industrialized nations. In the United States these have been classified as complimentary and alternative medicines (CAM) (National Center for Complementary and Alternative Medicine [NCCAM], 2000). Self help approaches are an integral part of these complimentary and alternative systems of medicine. Self-help approaches are very important for health education and health promotion as these can be used for preventive purposes in aiding development of health behaviors as well as useful adjuncts to several disease management programs.

In 1990 an estimated 60 million people used at least one alternative therapy which rose to 83 million people in 1997 (Eisenberg et al., 1998). In a national 2002 survey approximately 35\% of the population was found to be using at least one CAM therapy in the past year (Tindle, Davis, Phillips, \& Eisenberg, 2005). The same survey reported most commonly used CAM therapies were herbal therapy (18.6\%), relaxation techniques (14.2\%), and chiropractic (7.4\%).There are numerous reasons for this significant increase in the use of CAM. One of the suggested reasons has been the increase in the costs of health care and prescriptions (Jonas, 1998). Many people cannot afford the expensive therapies and want to try cheaper alternatives especially self-help approaches. People also want to increase their independence when illness occurs (Kaptchuk \& Eisenberg, 1998). Practicing self-help approaches often helps individuals feel as if they are taking control of their health and is an important reason for their popularity. Inability of conventional therapies to provide cures for chronic ailments, side effects and adverse effects with conventional therapies, 
and increased public awareness about unconventional therapies mainly through information technology has also enhanced their attractiveness (NCCAM, 2000).

The use of self-help approaches is increasing in older adults. Use of CAM therapies in older adults is of significant concern (Flaherty, \& Takahashi, 2004). Bruno and Ellis (2005) found that about $13 \%$ elderly over the age of 65 years had used an herbal supplement within the past 12 months. According to the U.S. Census Bureau's projections, by the year 2020 there will be 95,841,000 people over the age of 55 years (United States Census Bureau, 2001). When compared to the estimated 59,142,000 people over the age of 55 years in the year 2000, that is a $62 \%$ increase in the number of people 55 years and over (United States Census Bureau, 2001). It is projected that as the baby boomers become older there will be a significant increase in the number of older adults in the next twenty years (United States Census Bureau, 2001). This will increase the number of individuals requiring some type of medical care. If efficacy of these CAM therapies is established, then more health functionaries may be able to recommend CAM in treatment or prevention. A qualitative study in senior citizens found that they perceived CAM therapies to be extremely beneficial (Williamson, Fletcher, Dawson, 2003). There is little research on CAM therapies among older adults and in order to increase the validity of these approaches more research must be conducted. A growing number of studies are demonstrating efficacy of some CAM approaches particularly self-help approaches (Coker, 1999; Luskin et al., 2000; Pandya, Vyas, \& Vyas, 1999), but still further research is needed. The National Institutes of Health (NIH) has established a center and is allocating funds toward further research of CAM therapies (NCCAM, 2000). Finally, many of the complementary and alternative systems of health, such as self-help approaches emphasize more on prevention rather than cure and more on building healthy life styles which are more useful in health education and health promotion.

In the studies done about CAM in older adults differentiation between self-help approaches and approaches that depend on external agents, healers, or practitioners and are thus amenable to commercialism has not been addressed. Further, none of the studies done with older adults regarding CAM therapies have collected information on behavioral theory-based attitudes that predict or explain usage. Furthermore, selfhelp approaches are popular and low cost measures that can complement existing forms of therapy and improve coping with several chronic illnesses that are not possible to cure especially among older adults.

Therefore, this study aimed at understanding the prevalence and determinants of self-help approaches in older adults. The specific purposes of this study were to determine the usage, knowledge, costs, self-efficacy regarding use, and expectations regarding four commonly used health-related self-help approaches among older adults. The four health-related self-help approaches chosen were self-prayer, relaxation, yoga, and tai chi. The choice of these four approaches was made based upon the work of Eisenberg and colleagues (1998) that found these four approaches as most common self-help approaches. Another study by Dunn and Horgas (2000) in older adults also included these four self-help approaches. The social cognitive theory (Bandura, 1986, 2004) and stages of change model (Prochaska, Redding, \& Evers, 2002) were used as behavioral theories in designing the instrument for collecting data for this study.

\section{Methodology}

The population for this study consisted of the members enrolled in a voluntary health-related program for people over 55 years based in an urban setting in the Midwestern United States. The program is affiliated with a health system and enrolls anyone who is over the age of 55 years and wants to sign up. The health condition of the enrollee is not a criterion for membership. Membership benefits include receiving a quarterly newsletter about health issues and other information about the activities of the hospital and its outreach efforts. The program has a list of 10,338 members with most of the members belonging to the metropolitan area where the study was conducted. 
A questionnaire was developed and validated for face and content validity by four national experts in two rounds. The final instrument had six parts. The first part asked the respondents about how much they knew about each of the four selfhelp approaches on a scale of enough (3), somewhat (2), a little (1), not at all (0) and if they had used any of these approaches in the past 12 months. A summation of items on this part was done which yielded a possible score range of $0-12$. The second part asked about the expense on each of the approach in the past month. The third part was about the self-efficacy (Bandura, 1986, 2004) of performing each of the four self-help approaches on a scale of very sure (4), moderately sure (3), slightly sure (2) and not at all sure (1) with a range of $0-12$. The fourth part was about outcome expectations and outcome expectancies (Bandura, 1986, 2004). Outcome expectations are anticipatory benefits that a person has about outcomes of a behavior (Bandura, 1986, 2004). These are important for motivating a person to initiate specific behaviors. Outcome expectations that were chosen in the instrument comprised of improvement in health, feeling good about oneself, enhancement in social life, treatment of specific diseases, modifying one's habits, pain relief, and cutting costs and were measured on a scale of always (3), sometimes (2), hardly ever $(1)$, never $(0)$. Outcome expectancies are how much importance a person places on outcomes (Bandura, 1986, 2004). Hence, the value one placed on each of the outcome expectations was measured on a scale of very important (3), moderately important (2), slightly important (1), and not at all important (0). Outcome expectations were multiplied with outcome expectancies and were then summed to obtain a possible range of 0-63 units for expectations on this scale. The fifth part was about determining the present use of self-help approach based on the stages of change or transtheoretical model (Prochaska, Redding \& Evers, 2002). The question asked the respondents to indicate whether they had been regularly using a selfhelp approach for over six months (maintenance), less than six months (action), not regularly using (preparation), thinking about using (contemplation), or did not intend to use (pre-contemplation). The sixth section was about demographic information pertaining to gender, age, race, and education. The Flesch-Kincaid Reading ease of 57.2 and Flesch-Kincaid Grade level was found to be 7.8 and thus acceptable. Further the instrument was pilot tested for readability and comprehension with twelve members from the target population to test for readability and assess time taken to complete it before the administration. In pilot testing it was determined that no changes were needed.

Institutional Review Board (IRB) permission from the University was obtained for the study. The instrument was mailed to the 10,338 members. Sixty-two percent of these members are females and $38 \%$ are males as compared to the National US Census data of people over 55 years, which shows $51 \%$ females and $49 \%$ males. The number of participants in the ages 55-64 years in the membership is $39 \%$ as opposed to $41 \%$ nationally, in ages $65-74$ years $35 \%$ as opposed to $31 \%$ nationally and $26 \%$ as opposed to $28 \%$ nationally. A total of 2,726 people responded (26.4\% response rate). A total of 2,119 responded within two weeks and then a reminder was mailed and another 607 responded. A chi-square analysis was performed between these two groups and no significant difference was found in any study variable. This approach of analysis that compares first time responders with second time responders is based on the work of Little and Rubin (1987) and Oh and Schuren (1983) that assumes that the second time responders share the characteristics with the non-responders. If no differences are found, one can assume that the first time responders and non-responders are similar in characteristics and that the role of non-response bias may be minimal. All data were analyzed using SPSS for Windows, Version 10 (1999). For differentiating pre-contemplators or contemplators from those that were in the preparation, action, or maintenance stage logistic regression was done.

\section{Results}

The total sample consisted of $67.0 \%$ females $(n=1,820)$ and $33.0 \%$ males $(n=895)$. Thirtyseven percent $(n=1,008)$ of the respondents were between 55-64 years, 38.8\% were between the ages of 65 and $74(n=1,053)$, and $24.1 \%$ over 75 years $(n=654)$. The sample was 
predominantly White as expected in the Midwest ( $n=2,590,97.5 \%)$. Only 106 (4.3\%) respondents had less than high school education and 1,687 (61.8\%) had completed college education or more. The main differences in the sample with the national data with regard to demographics pertain to the larger proportion of women, exclusive representation of White race, and higher educational qualifications.

Table 1 summarizes the descriptive findings from the study. Self-prayer was reported as the most common self-help approach with 60\% having used it within the past year. Twenty-three (23) percent of the respondents reported the use of relaxation. Approximately six (6) percent reported the use of yoga and tai chi each. Overall, $61.6 \%$ of respondents utilized at least one form of self-help approach.

The study also identified that a mean of US \$2.33 was spent out-of-pocket per month on self-help approaches. Out-of-pocket expense refers to the co-payment or deductible payment that is made by the patient and is not covered by the insurance company. Perceived knowledge about these approaches was measured on a scale of not at all (0), a little (1), somewhat (2), enough (3) and the scores were summed (0-12 units). The mean score was 4.46 units. The mean scores for self-prayer (1.82) and relaxation (1.14) were in the middle of the possible range $(0-3)$ but yoga $(0.85)$ and tai chi $(0.66)$ were on the lower end.

The mean expectation score for self-help approaches was found to be 32.18 units or in the middle of the possible range of 0-63. The mean self-efficacy score was found to be 4.39 units on a possible range of $0-12$ units. Using the transtheoretical model described by Porchaska and colleagues (2002), the respondents were classified into the five stages of precontemplation, contemplation, preparation, action, and maintenance regarding self-help approaches. Only 28 percent were not thinking about using these (pre-contemplators) and a majority $(44.6 \%)$ reported they were in the maintenance stage.

Logistic regression modeling was done to identify significant predictors that differentiated pre-contemplators or contemplators from those that were in the preparation, action, or maintenance stage. The results are depicted in Table 2. Significant predictors were found to be knowledge, self-efficacy in performing self-help approaches and expectations about self-help approaches.

\section{Discussion}

More than three-fifths (61.6\%) of respondents utilized at least one form of self-help approach thereby implying the popularity of these approaches among older adults. These results are in consonance with the findings of Eisenberg and colleagues (1998) which found that the most popular self-help approach was self-prayer. This is also in consonance with the findings from the study done by Dunn and Horgas (2000) which found that a large proportion of older adults mentioned using self-prayer and relaxation (82\%) while yoga and tai chi were not as prevalent. This is not surprising because prayer is deep rooted in the Judeo-Christian culture that has predominant influence in the region from where the sample was drawn. Relaxation is also advocated by many mainstream physicians and is gaining popularity. While yoga and tai chi are Asian concepts and as such not that prevalent but seem to be gaining more importance. The reported average monthly cost of US $\$ 2.33$ on self-help approaches which is minuscule. Hence, self-help approaches are both popular and offer potential for popularization among older adults as close to two-fifths (38.4\%) of older adults are in pre-contemplation or contemplation stages with regard to applying self-help approaches. 
Table 1

Descriptive statistics of prevalence, costs, and behavioral variables $(n=2,726)$

\begin{tabular}{|l|l|c|c|c|}
\hline \multicolumn{1}{|c|}{ Variable } & \multicolumn{1}{|c|}{ Categories } & Valid n & Frequency (\%) & Mean (s.d.) \\
\hline Prevalence & Self-prayer & 2,545 & $1,524(59.9)$ & \\
\hline & Relaxation & 2,538 & $581(22.9)$ & \\
\hline & Yoga & 2,532 & $165(6.1)$ & \\
\hline $\begin{array}{l}\text { Out-of-pocket costs per month } \\
\text { on self-help approaches (in \$) }\end{array}$ & Tai Chi & 2,524 & $158(5.8)$ & \\
\hline Perceived knowledge (0-12) & & 2,358 & & $2.33(18.90)$ \\
\hline & Self-prayer (0-3) & 2,514 & & $4.46(3.03)$ \\
\hline & Relaxation (0-3) & 2,585 & & $1.82(1.12)$ \\
\hline & Yoga (0-3) & 2,605 & & $1.14(1.00)$ \\
\hline & Tai Chi (0-3) & 2,599 & & $0.85(0.91)$ \\
\hline Self-efficacy (0-12) & & 2,504 & & $0.66(0.89)$ \\
\hline Expectations (0-63) & & 2,189 & & $32.18(3.12)$ \\
\hline Stage of change & & 2,561 & & \\
\hline & Pre-contemplation & & $721(28.2)$ & \\
\hline & Contemplation & & $260(10.2)$ & \\
\hline & Preparation & & $343(13.4)$ & \\
\hline & Action & & $94(3.7)$ & \\
\hline & Maintenance & & $1,143(44.6)$ & \\
\hline
\end{tabular}

Table 2

Analysis of maximum likelihood estimates of study variables for older adults in active or maintenance stage of using self-help approaches versus pre-contemplation, contemplation, or preparation stages

\begin{tabular}{|l|l|c|c|c|c|c|c|}
\hline \multicolumn{1}{|c|}{ Variable } & df & $\begin{array}{c}\text { Parameter } \\
\text { Estimate }\end{array}$ & $\begin{array}{c}\text { Std. } \\
\text { Error }\end{array}$ & $\begin{array}{c}\text { Wald's } \\
\text { Chi-square }\end{array}$ & p-value & $\begin{array}{c}\text { Odds } \\
\text { Ratio }\end{array}$ & $\begin{array}{c}\text { 95\% CI } \\
\text { of OR }\end{array}$ \\
\hline Intercept & 1 & -2.6183 & & & & & \\
\hline Knowledge & 1 & 0.2091 & 0.0223 & 88.1252 & 0.0001 & 1.23 & $(1.18,1.29)$ \\
\hline Self-efficacy & 1 & 0.0829 & 0.0216 & 14.7123 & 0.0001 & 1.09 & $(1.04,1.13)$ \\
\hline Expectations & 1 & 0.0350 & 0.0044 & 63.9531 & 0.0001 & 1.04 & $(1.03,1.04)$ \\
\hline Monthly cost & 1 & 0.0097 & 0.0060 & 2.5830 & 0.1080 & 1.01 & $(0.99,1.02)$ \\
\hline
\end{tabular}

Using logistic regression modeling it was demonstrated that knowledge (behavioral capability) about self-help approaches, expectations about self-help approaches and self-efficacy about self-help approaches, were significant predictors for being in preparation, action or maintenance stages. All these constructs are modifiable. These constructs are from the social cognitive theory (Bandura, 1986, 2004) and quite popular in behavior change programs. The knowledge and self-efficacy scores were found to be around the middle of the range implying that older adults do not have high levels of confidence in their ability to use these techniques. The study supports the findings that self-help approaches, particularly self-prayer and relaxation, are commonly used by older adults, these are not expensive and offer the potential to complement existing therapies thereby enhancing coping in older adults.

The study has several limitations. Firstly, the sample was not randomly selected resulting in sampling bias. Strictly speaking, the results are primarily applicable to urban older adults in Midwestern United States. Secondly, the 
response rate was also less than $30 \%$ resulting in non-response bias. A log was not kept of respondents who did not participate in the study and efforts were not made to contact them (nonresponders). However a comparison between first time respondents and second time respondents was done and no differences were found. Assuming that there were no differences between second time responders and nonrespondents it can be said that the influence of non-response bias was minimal. Thirdly, the respondents were asked to recall their behaviors over a period of past 12 months and that is difficult especially for some older adults and leads to recall bias. Finally, the design of the study was cross-sectional and, therefore, nothing can be said about the temporality of association. Despite these limitations the study provides some useful results.

\section{Implications for Health Education and Health Promotion}

It is evident from this study that more health education and health promotion programs that promote self-help approaches in older adults are needed. The self-help approaches can include self-prayer, relaxation, yoga and tai chi. In order to build these self-help approaches behavioral theories such as social cognitive theory and stages of change model can be utilized. Behavioral capability or knowledge is an essential component in initiation of behavior change and found to be a significant in this study. Behavioral capability can be built by health education programs utilizing lectures, skill building workshops, developing brochures, pamphlets, and web-based information systems.

Expectations about self-help approaches were found to be another significant predictor in this study. This can be built by health education programs that emphasize the benefits of selfhelp approaches and help the older adults imbibe those benefits at a personal level. Educational methods such as group discussions, brainstorming, role plays, and simulations can be used in this regard.

Self-efficacy for self-help approaches which was another significant construct that can be built through using credible older adult role models. Self-efficacy can be further created by focusing on specific tasks that need to be done in selfhelp approaches, breaking down the complex tasks into smaller steps, and providing opportunities for participatory practice that leads to mastery. Self-help approaches are popular among older adults but the programs that help older adults systematically learn these approaches are deficient. Hence, in conclusion, it can be said that more needs to be done with regard to understanding the determinants of selfhelp approaches in older adults and designing and evaluating effective interventions.

\section{References}

Bandura, A. (1986). Social foundations of thought and action. Englewood Cliffs, NJ: Prentice Hall.

Bandura, A. (2004). Health promotion by social cognitive means. Health Education \& Behavior, 31, 143164.

Bruno, J. J., \& Ellis, J. J. (2005). Herbal use among US elderly: 2002 national health interview survey. The Annals of Pharmacotherapy, 39, 643-648.

Coker, K. H. (1999). Meditation and prostate cancer: Integrating a mind/body intervention with traditional therapies. Seminar in Urologic Oncology, 17, 111-118.

Dunn, K. S., \& Horgas, A. L. (2000). The prevalence of prayer as a spiritual self-care modality in elders. Journal of Holistic Nursing, 18, 337-351.

Eisenberg, D., Davis, R., Ettner, S., Appel, S., Wilkey, S., Rompay, M. et al. (1998). Trends in alternative medicine use in the United States 1990-1997. Journal of the American Medical Association, 280, 1569-1575.

Flaherty, J. H., \& Takahashi, R. (2004). The use of complementary and alternative medical therapies among older persons around the world. Clinics in Geriatric Medicine, 20, 179-200.

Jonas, W. (1998). Alternative medicine. Learning from the past, examining the present, advancing to the future. Journal of American Medicine, 280, 1616-1618. 
Kaptchuk, T. J., \& Eisenberg, D. M. (1998). The persuasive appeal of alternative medicine. Annals of Internal Medicine, 129, 1061-1065.

Little, R. J. A., \& Rubin, D. B. (1987). Statistical analysis with missing data. New York: John Wiley and Sons.

Oh, H. L., \& Schuern, F. S. (1983). Weighting adjustments for unit non response. In W. G. Madow, I. Olkin, \& D. B. Rubin (Eds.), Incomplete data in sample surveys. Volume II: Theory and annotated bibliography. New York: Academic Press.

Luskin, F. M., Newell, K. A., Griffith, M., Holmes, M., Telles. S., DiNucci, E. et al. (2000). A review of mind/body therapies in the treatment of musculoskeletal disorders with implications for the elderly. Alternative Therapies in Health and Medicine, 6, 46-56.

Tindle, H. A., Davis, R. B., Phillips, R. S., \& Eisenberg, D. M. (2005). Trends in use of complementary and alternative medicine by US adults: 1997-2002. Alternative Therapies in Health and Medicine, $11,42-49$.

National Center for Complementary and Alternative Medicine. (2000). Expanding horizons of health care. Five year strategic plan 2001-2005. Gaithersburg, MD: NCAAM Clearinghouse.

Pandya, D. P., Vyas, V.H., \& Vyas, S. H. (1999). Mind-body therapy in the management and prevention of coronary disease. Comprehensive Therapy, 25, 283-293.

Prochaska, J. O., Redding, C. A., \& Evers, K. E. (2002). The transtheoretical model and stages of change. In K. Glanz, B. K. Rimer, \& F. M. Lewis (Eds.), Health behavior and health education. Theory, research and practice. (3rd ed., pp. 99-120). San Francisco, CA: Jossey Bass, Inc.

Statistical Package for Social Sciences (SPSS). (1999). Statistical package for social sciences (SPSS) (Version 10). [Computer software]. Chicago, IL: Author.

United States Census Bureau. (2001). Resident population estimates of the United States by age and sex. (2001). Retrieved November 12, 2005, from http://www.census.gov/population/estimates/nation/intfile2-1.txt

Williamson, A. T., Fletcher, P. C., \& Dawson, K. A. (2003). Complementary and alternative medicine. Use in an older population. Journal of Gerontological Nursing, 29, 20-28.

\author{
Author Information \\ Angela Drent, MS \\ Community Networking / 55Plus \\ Methodist Health System \\ 8303 Dodge St \\ Omaha, NE 68114 \\ Ph.: 402-354-872 \\ E-Mail: Angela.Drent@nmhs.org \\ Manoj Sharma, MBBS, CHES, Ph.D.* \\ Associate Professor \& \\ Masters Thesis Chair at University of Nebraska at Omaha \\ Health Promotion \& Education \\ University of Cincinnati \\ 526 Teachers College \\ PO Box 210002 \\ Cincinnati, OH 45221-0002 \\ Ph.: 513-556-3878 \\ Fax.:513-556-3898 \\ E-Mail: manoj.sharma@uc.edu \\ *Corresponding Author
}

